\title{
A FUNDAMENTAL THEOREM ON DECOMPOSITIONS OF THE SPHERE INTO POINTS AND TAME ARCS
}

\author{
KYUNG WHAN KWUN
}

1. Introduction. R. H. Bing showed that an upper semicontinuous decomposition of $E^{3}$ into points and tame arcs did not necessarily produce a decomposition space topologically equivalent to $E^{3}$ [1]. M. L. Curtis and R. L. Wilder showed [2] that the decomposition space of Bing was a homotopy manifold, thus negatively answering a question proposed by Griffiths [3]. R. Rosen recently announced [4] a result which in part appears to be an example of $E^{3}$ into points and tame arcs such that the decomposition space is not locally euclidean at any point. Thus, upper semicontinuous decompositions of $E^{3}$ (or $S^{3}$ ) into points and tame arcs yield various types of decomposition spaces. It is the purpose of this paper to prove a theorem which in particular implies that every upper semicontinuous decomposition of $S^{3}$ into points and tame arcs yields a homotopy manifold decomposition space. Arguments employed here are completely elementary.

2. Some lemmas. Let $R_{i}, i=1,2$, be the sphere in $E^{n}$ of radius $i$ with the center at the origin. We denote by $O_{i}$ the interior of $R_{i}$ in $E^{n}$. Let $U$ be an open $n$-cell containing another open $n$-cell $V$ and $x$ a point of $V$. We say that $V$ is concentric with $U$ or that $U$ and $V$ are concentric if and only if there is a homeomorphism of $U$ onto $O_{2}$ which sends $V$ onto $O_{1}$. We observe that the homeomorphism can be chosen so as to map $x$ to the origin.

Lemma 1. Let $U$ be an open $n$-cell and $V$ and $W$ open subsets of $U$ such that $W \subset V \subset U$ and $W$ is an open n-cell concentric with $U$. Let $x$ be a point of $W$. Let $i$ and $i^{\prime}$ be the inclusion maps of $W-x$ into $V-x$ and of $V-x$ into $U-x$, respectively. Then the induced homomorphisms $i_{*}$ and $i_{*}^{\prime}$ of homotopy groups are injective and surjective, respectively.

Proof. We observe that $i_{*}^{\prime} \cdot i_{*}=\left(i^{\prime} \cdot i\right)_{*}$ is bijective. The homomorphism $i_{*}$ is injective, since $i_{*}^{\prime} \cdot i_{*}$ is, and $i_{*}^{\prime}$ is surjective, since $i_{*}^{\prime} \cdot i_{*}$ is.

Lemma 2. Letf be a proper map of a Hausdorff space $X$ onto a locally compact Hausdorff space $Y$. Let $y$ be any point of $Y$ and $U$ be any neighborhood of $A=f^{-1}(y)$. Then there exists a neighborhood $V$ of $y$ such that $f^{-1}(V) \subset U$. 1960

Received by the editors December 14, 1959, and, in revised form, February 6, 
We recall that a proper map is a continuous map such that the inverse image of each compact set is compact.

PROOF OF THE LEMMA. Let $v$ be the family of compact neighborhoods of $y$. Then each $f^{-1}\left(V_{\alpha}\right), V_{\alpha} \in \mathcal{V}$, is compact, and $\cap f^{-1}\left(V_{\alpha}\right)=A$, or $\cap f^{-1}\left(V_{\alpha}\right) \cap(X-U)=\varnothing$, so that there is a finite family $V_{1}, V_{2}, \cdots$, with $\cap f^{-1}\left(V_{i}\right) \subset U$. We now take $V=\cap V_{i}$.

The author is indebted to Professor Hans Samelson for strengthening Lemma 2 to the present form.

\section{The main theorem.}

MAIN THEOREM. Let $G$ be an upper semicontinuous decomposition of $S^{n}$ with a finite dimensional decomposition space $X$. If each element $g$ of $G$ is an absolute retract having an open $n$-cell as complement in $S^{n}$, then $X$ is an n-dimensional homotopy manifold.

PROOF. Since each $g \in G$ is contractible, Wilder's mapping theorem $[6 ; 7]$ applies to show that $X$ is an $n$-gcm. Since each $g \in G$ is locally contractible and homotopically trivial, by Smale's theorem [5], $X$ is $\mathrm{LC}^{\infty}$. There remains to show that $X$ has the same local homotopy character as $S^{n}$, i.e., for given $x \in X$ and a neighborhood $U$ of $x$, there exist neighborhoods $V$ and $W$ of $x$ such that $U \supset V \supset W$ and the image of $\pi_{k}(W-x)$ in $\pi_{k}(V-x)$ under the inclusion map is isomorphic to $\pi_{k}\left(S^{n-1}\right)$.

Let $G_{1}$ be the upper semicontinuous decomposition of $S^{n}$ consisting of $g_{1}=f^{-1}(x)$ and the points not on $g_{1}$, where $f$ is the quotient map of $G$. We denote by $X_{1}$ and $f_{1}$ respectively the decomposition space and the quotient map of $G_{1}$. Let $G_{2}$ be the upper semicontinuous decomposition of $X_{1}$ consisting of $f_{1}(g), g \in G$. We denote by $X_{2}$ and $f_{2}$ the decomposition space and the quotient map of $G_{2}$, respectively. By assumption, $X_{1}$ is a topological $n$-sphere. It is easy to see that $X_{2}=X$ and $f=f_{2} \cdot f_{1}$ after the natural identification. By construction, $x_{1}=f_{2}^{-1}(x)$ is a point of $X_{1}$. Let $U_{1}=f_{2}^{-1}(U)$. Since $X_{1}$ is a manifold, there exists an open $n$-cell neighborhood $U_{1}^{\prime}$ of $x_{1}$ contained in $U_{1}$. By Lemma 2, there exists a neighborhood $V$ of $x$ such that $f_{2}^{-1}(V) \subset U_{1}^{\prime}$. There exists an open $n$-cell neighborhood $U_{1}^{\prime \prime}$ of $x_{1}$ contained in $V_{1}=f_{2}^{-1}(V)$ such that $U_{1}^{\prime \prime}$ is concentric with $U_{1}^{\prime}$. By Lemma 2 again, there exists a neighborhood $W$ of $x$ such that $W_{1}=f_{2}^{-1}(W) \subset U_{1}^{\prime \prime}$. There exists an open $n$-cell neighborhood $U_{1}^{\prime \prime \prime}$ of $x_{1}$ such that $U_{1}^{\prime \prime \prime}$ is contained in $W_{1}$ and is concentric with $U_{1}^{\prime \prime}$.

Let the letters above the arrows in the following diagram denote the appropriate inclusion maps. 


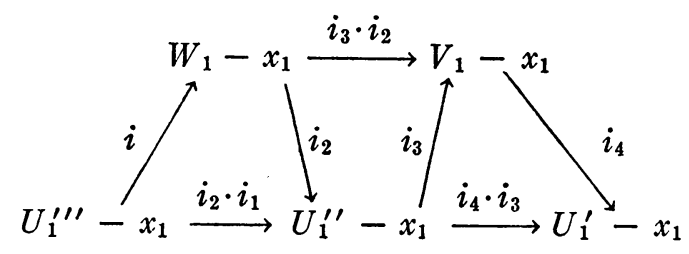

The starred letters will denote the homomorphisms of homotopy groups induced by the corresponding inclusion maps. By Lemma 1, the homomorphisms $i_{2 *}$ and $i_{3 *}$ are surjective and injective, respectively. Hence, considering the fact that $U^{\prime \prime}-x_{1}$ is of the same homotopy type as $S^{n-1},\left(i_{3} \cdot i_{2}\right)_{*} \pi_{k}\left(W_{1}-x_{1}\right)=i_{3 *} \pi_{k}\left(U_{1}^{\prime \prime}-x_{1}\right) \simeq \pi_{k}\left(U_{1}^{\prime \prime}-x_{1}\right)$ $\simeq \pi_{k}\left(S^{n-1}\right)$.

Now we denote by $i$ and $i^{\prime}$ respectively the inclusion maps of $W_{1}-x_{1}$ into $V_{1}-x_{1}$ and of $W-x$ into $V-x$. Clearly, $f_{2} \cdot i=i^{\prime} \cdot f_{2}$. Hence, the following diagram is commutative.

$$
\begin{array}{cc}
\pi_{k}\left(W_{1}-x_{1}\right) & \stackrel{i_{*}}{\longrightarrow} \pi_{k}\left(V_{1}-x_{1}\right) \\
\downarrow f_{2 *} & \stackrel{i_{*}^{\prime}}{\longrightarrow} \pi_{k}(V-x) .
\end{array}
$$

By the already-mentioned Smale's theorem again, the $f_{2 *}$ are bijective. Hence,

$$
\begin{aligned}
i_{*}^{\prime} \pi_{k}(W-x) & =i_{*}^{\prime} \cdot f_{2 *} \pi_{k}\left(W_{1}-x_{1}\right)=f_{2 *} \cdot i_{*} \pi_{k}\left(W_{1}-x_{1}\right) \\
& \simeq i_{*} \pi_{k}\left(W_{1}-x_{1}\right) \simeq \pi_{k}\left(S^{n-1}\right)
\end{aligned}
$$

4. Some remarks. The absolute retract condition in the statement of the main theorem is much stronger than necessary. (See [5].) The space $S^{n}$ can be replaced by any closed manifold by requiring that the space minus $g$ is homeomorphic with a closed manifold minus a point. Also a similar conclusion can be drawn for open manifolds if we impose some restrictions on $f$. Thus various forms of generalizations are available at the price of simplicity. On the other hand, the main theorem cannot be generalized much further. For instance, the condition that $S^{n}-g$ be an open $n$-cell cannot be deleted. In fact, it cannot be replaced by the condition that $S^{n}-g$ be simply connected. (See (3) of Part II of [2].)

R. Rosen's example [4] of a totally noneuclidean cartesian factor of $E^{4}$ is nevertheless a homotopy manifold. Professor M. Curtis points out that there is a cartesian factor of $E^{4}$ which is not a homotopy 
manifold. Thus a cartesian factor of a homotopy manifold is not necessarily a homotopy manifold.

\section{REFERENCES}

1. R. Bing, $A$ decomposition of $E^{3}$ into points and tame arcs such that the decomposition space is topologically different from $E^{3}$, Ann. of Math. vol. 65 (1957).

2. M. Curtis and R. Wilder, The existence of certain types of manifolds, Trans. Amer. Math. Soc. vol. 91 (1959) pp. 152-160.

3. H. Griffiths, $A$ contribution to the theory of manifolds, Michigan Math. J. vol. 2 (1953) pp. 61-89.

4. R. Roxen, $E^{4}$ is the Cartesian product of a totally non-euclidean space and $E^{1}$, Notices Amer. Math. Soc., Abstr. 563-1, vol. 6 (1959) p. 641.

5. S. Smale, A Vietoris mapping theorem for homotopy, Proc. Amer. Math. Soc. vol. 8 (1957) pp. 604-610.

6. R. Wilder, Monotone mappings of manifolds, Pacific J. Math. vol. 3 (1957) pp. 1519-1528.

7. - Monotone mappings of manifolds II, Michigan Math. J. vol. 5 (1958) pp. 19-25.

The Seoul National University, Seoul, Korea

\section{LOGARITHMICALLY EXACT DIFFERENTIAL FORMS}

\section{RICHARD S. PALAIS ${ }^{1}$}

Let $M$ be a connected, differentiable $\left(=C^{\infty}\right)$ manifold. Let $C^{1}(M, C)$ denote the complex vector space of complex valued one-forms on $M$ : an element $\omega$ of $C^{1}(M, C)$ is a function which assigns to each $x \in M$ a linear map $\omega_{x}$ of $M_{x}$ (the tangent space to $M$ at $x$, a real vector space) into the complex numbers $C$, such that if $X$ is a differentiable vector field on $M$ then $x \rightarrow \omega_{x}\left(X_{x}\right)$ is a differentiable complex valued function on $M$. Each element $\omega$ of $C^{1}(M, C)$ can be written uniquely in the form $\mu+i \nu$ where $\mu$ and $\nu$ are real valued one-forms on $M$, and we put $\mu=\operatorname{Re} \omega$ and $i \nu=\operatorname{Im} \omega$. We write $Z^{1}(M, C)$ for the subspace of $C^{1}(M, C)$ consisting of closed forms and $B^{1}(M, C)$ for the subspace of $Z^{1}(M, C)$ consisting of exact forms.

An element of $C^{1}(M, C)$ will be called logarithmically exact if it is of the form $d f / f$ for some nowhere vanishing, differentiable, complex valued function $f$ on $M$. Since $d(d f / f)=\left(f d^{2} f-d f \wedge d f\right) / f^{2}=0$ and $d f / f-d g / g=d(f / g) /(f / g)$ it is clear that the set $L^{1}(M, C)$ of logarithmically exact one-forms is a subgroup (but not in general a subspace)

Received by the editors February 24, 1960.

1 The author is a National Science Foundation postdoctoral fellow. 